\title{
A Practical Data Processing Analysis for Water Impact Problem
}

\author{
WANG Yonghu \\ Civil Aviation Flight University of China, Guanghan, Sichuan Province, China, 618307 \\ wangyh.cn@gmail.com
}

\begin{abstract}
Keywords: Water Impact; Data assessment; Filtering; Cutoff frequency; Low-pass filter. Abstract: The paper presents a practical data processing methodology to smooth away high frequency noise for water impact problem. At first, original experimental data analysis is employed to design the real time low-pass filter, especially for the cutoff frequency of the filter. At the same time, velocity-time histories based on integration of acceleration signal are utilized to carry out the data assessment. Moreover, the desired cut-off frequency is selected in the pulse checking segment, comparing the velocity or displacement time histories between the original and the reconstructed pulses. Finally, it is helpful to design a desired low-pass filter used to post-process acceleration data and implement the simulation model validation.
\end{abstract}

\section{Introduction}

It is challenging to obtain and process the dynamic test data in the experimental mechanics. Test data obtained always include the actual physical signal and electrical noise. It is always imposed on the acceleration-time data which is the most important for carsh dyanmics. This paper aims at removing the noise from the whole signal in order to improve the signal-to-noise ratio (SNR) and it provids us some good information. The magnitude and duration of the low frequency signal are often concerned in impact dynamics and one is of little interested in the high-frequency signal [1, 2].

It is general method to average all measurement data because the true physical signal is substantially constant, and the noise is random. The noise must be eliminated by the average method which the limitation is more experimental time-needed and funds-investment. One can not ensure whether the test results can generate the required signals, then this method is the only way and the best one. Another method is the FFT filtering mehtod in which the frequency distribution can be acknowledged to determine a cutoff frequency through the frequency domain analysis. The high frequency signal will be removed due to the cutoff frequecy as a threshold, and then the ture physical signal is leaving. In fact, the trend of the signal is relatively clear, so the filtering methods will be used to process the raw data of the impacting test for the requirment of project. The outcoming characteristic is to remove spikes and retain the original physicial curve and its change trend.

\section{Data Assessment}

The test data should be checked for the quality assessment in order to ensure it as accurate as possible and later it will be used for simulation model validation. According to the filtering theory, some noises will be removed and the remain noise will still mask the real physical data. And some real physical data will filter out if the given cutoff frequency is bigger. They all will effect the actual impact load curve, especially the peak of crash response. In view of this consideration, data assessment must be carried out in advance.

If acceleration-time histories have anomalous signals, then the velocity obtained will be corrupted. Here, the data assessment is employed through ingrating acceleration data to produce velocity cure, as shown in Fig.1. In the process of water impact experiment, the whole sampling data can be divided into two sets including airborne segment and water impact segment. The second set of data is of concerned here and the duration of data assessement is from position A to position B of the velocity-time history. The signal period is computated by the maximum value (position $\mathrm{C}$ ) and the minimum value (position A), and the cutoff frequency will be obtained as the input parameter for the low-pass filiter required. But this cutoff frequency computed is obviously smaller in the pluse checking segment. In order to 
improve the quality of data processing, the maximum slop of the velocity-time cruve is computated here, and it is equal to the maximum acceleration which is the important value for water impact problem.

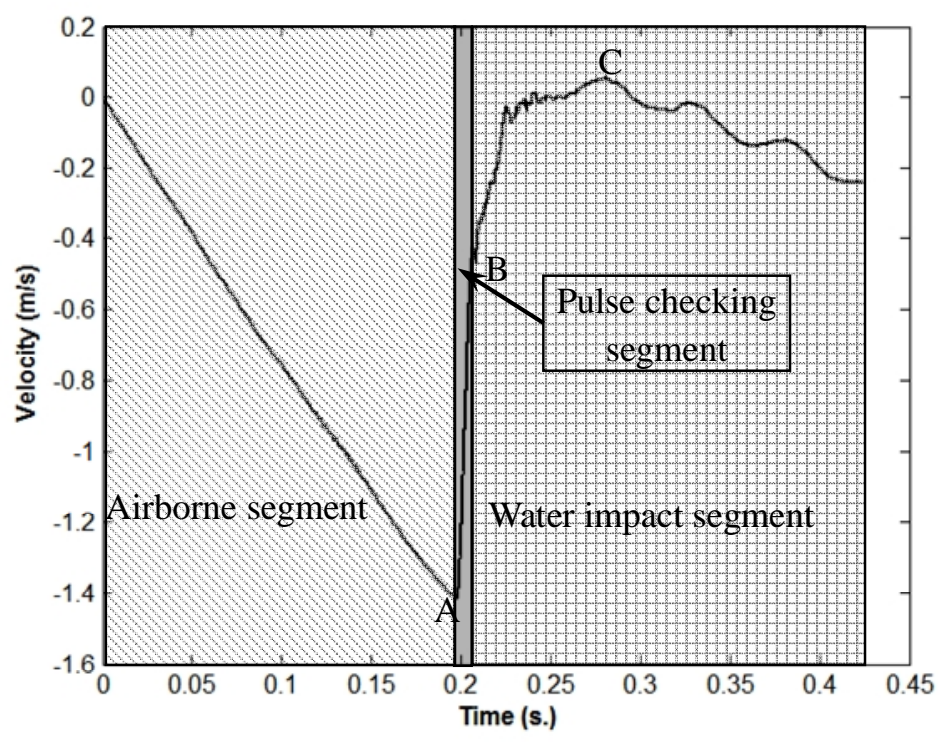

Fig 1. Velocity-time history of water impact

According to the trend of the velocity-time curve, the maximum acceleration is approximated by the average acceleration. The average value is the change in velocity divided by the time interval as shown below:

$$
\bar{A}=\frac{V_{f}-V_{i}}{T_{f}-T_{i}}
$$

where $V_{f}$ is the final velocity, $V_{i}$ is the initial velocity which equals to $\sqrt{2 g h}, T_{f}$ is the final time, and $T_{i}$ is the inital time. $\left[T_{i}, T_{j}\right]$ is the time inteval for the signal equality assessment and the cutoff frequency can be deterimined to design the desired filter.

\section{Real-time filter design}

The low-pass Butterworth digital filter used to post-process acceleration data is designed based on the general algorithm in the standard of SAE 211/1 [3] and the algorthm mentioned before. A set of Channel Frequency Classes (CFC) is selected blindly in water impact dynamics because the algorithm is appropriate for the impacts of vehicles. The correct low-pass filtering frequency should be deterimined from measuring the duration of the acceration pulse. Thus, the same cut-off frequency should not be used in all impacting events of millisecond level. Since a Butterworth low-pass filter has a relatively relaxing low-pass filtering characteristics, it is the key point for the filter to determining its cut-off frequency.

The cutoff frequecy is easily choosed through the FFT signal processing results [4]. Frequecny response curve is given as shown in Fig 2. And Fig 3 shows the comparison of orignal acceleration-time history and the filtered cruve by the low-pass filter. 


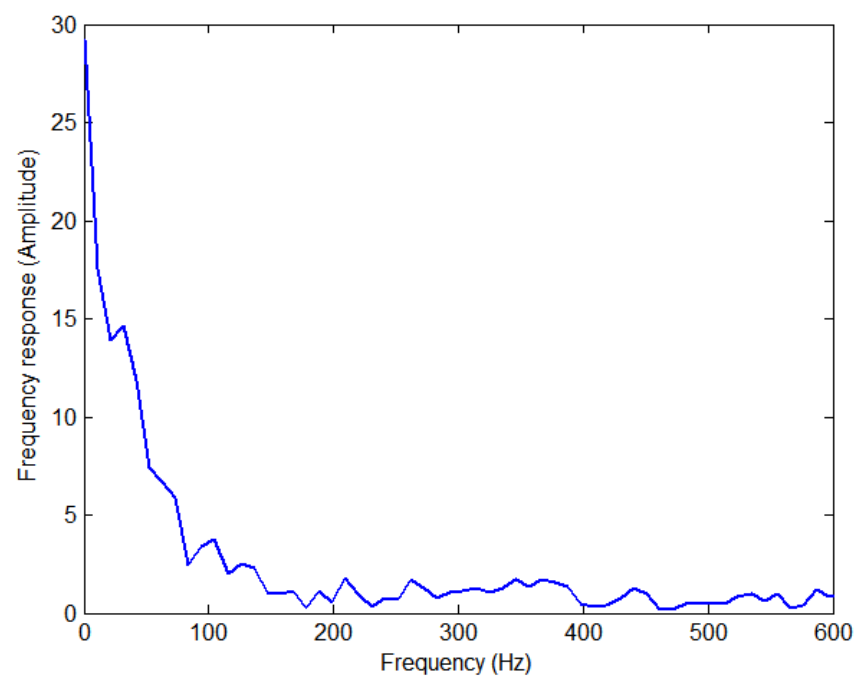

Fig 2. Frequency response curve

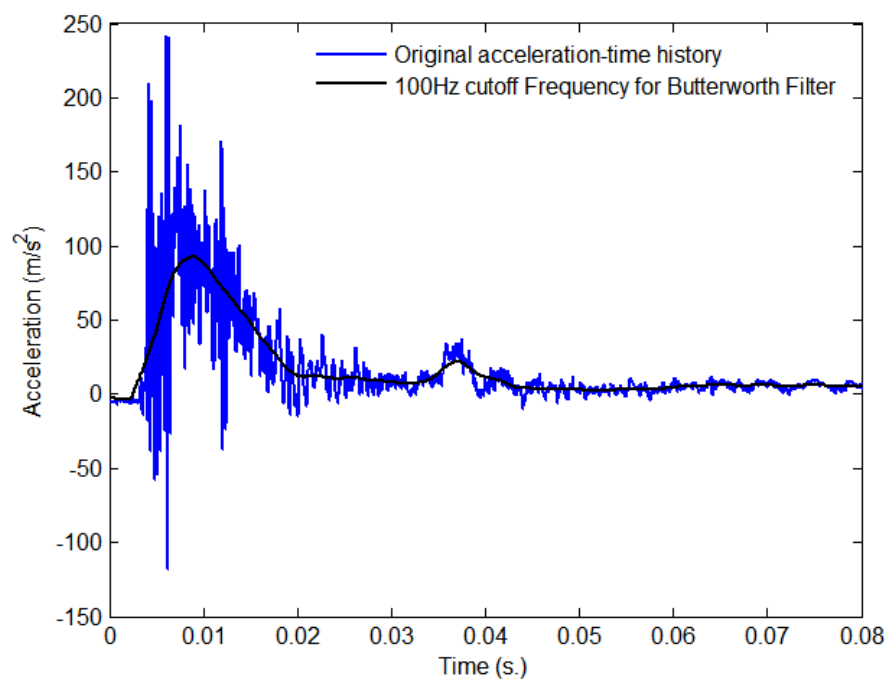

Fig 3. Filtering results by Butterworth filter $(100 \mathrm{~Hz})$

The processing results indicate that the low frequency band dominates the crash pulse and the cutoff frequency is chosen as $100 \mathrm{~Hz}$ which corresponds to the filter class CFC60. The velocity and displacement response obtained by integrating the filtered acceleration curve and velocity curve are shown in Fig 4 and Fig 5, respectively. From the figures, it is evident that they are followed quite well.

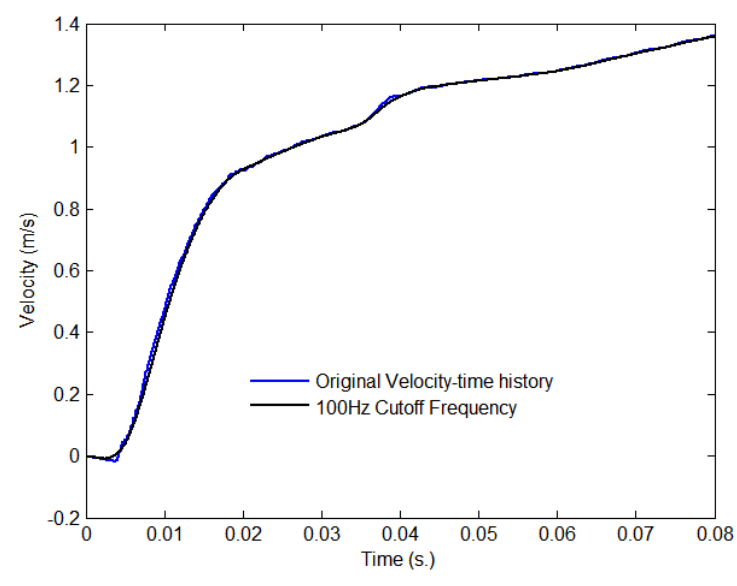

Fig 4. Comparison of velocity-time histories

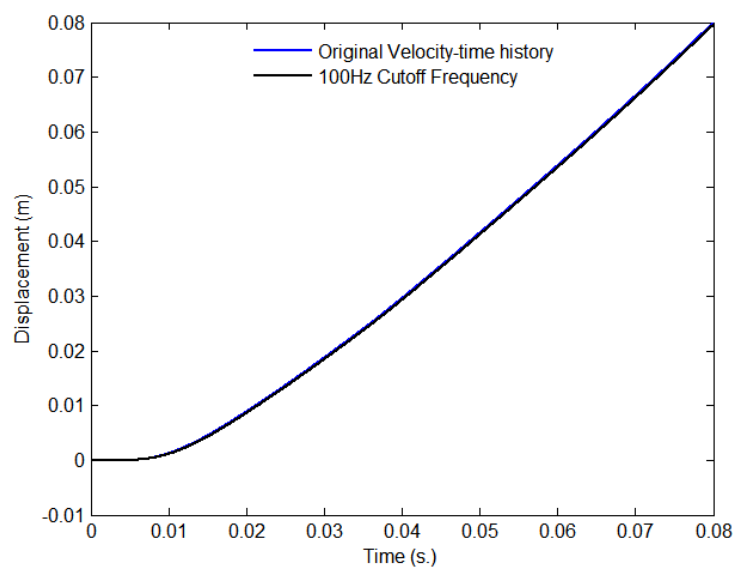

Fig 5. Comparison of displacement-time histories 
In the future, the low-pass filter with the $100 \mathrm{~Hz}$ cutoff frequency is effective enough to process the crash experimental data and meet the need of crash engineering. If the ideal filtered curves will be obtained by the in-depth analysis based on the analytical results and accurate test results, especially deep study of some key parameters of the filter. Generally speaking, it is feasible for data quality assessment that data from the acceleration response should be integrated to produce the velocity response. And the appropriate filter is determined based on different simulation conditions in accordance with the condition of impacting onto water.

In the process of the simulation, four filters given are generally to be selectd to filter simualtion data, named SAE, BW, FIR100 and COS filter. In general, BW and FIR100 filters are more appropriate selections for the later simulated data processing. For acceleration signals CFC60 filter is used and CFC180 filter for pressure data. The filters are selected by the methods mentioned before and some considerations.

\section{Conclusions and remarks}

In the reponse of crash dyanmics, the low frequency band dominates the crash pulse, as evidenced by the excitation of water impact. In order to smooth away the high frequency niose, the valuable discovery from the filtered pulse will be obtained in the good signal processing method. And the appropriate filter is designed and selected by oneself besed on the quality inspection and evaluation from crash responses. Velocity data integrated from the acceleration response are used to carry out data quality assessment. Here average acceleration over the time interval in data checking segment is computed because it is close to the maximum acceleration value. The Butterworth digital filter is designed based on the data assessment analysis, wherein the selected cut-off frequency is various with the differnet operation conditions.

\section{Acknowledgements}

This work was financially supported by the Joint Funds of the National Natural Science Foundation of China (U1333133), the General Program of CAFUC (J2014-04) and the Open Fund Program of Flight Technology and Flight safety research base (F2013KF02).

\section{References}

[1] M. Anghileri, et al. Rigid body water impact-experimental tests and numerical simulation using the SPH method. International Journal of Impact Engineering, Vol.38 (2011), P141-151.

[2] A. Grimaldi. Steel structure impacting onto water: coupled finite element-smoothed-particlehydrodynamics numerical modeling. Journal of Aircraft, Vol.48, No.4 (2011), P1299-1308.

[3] Society of Automotive Engineers. Surface Vehicle Recommended Practice: Instrumentation for Impact Test - Part 1, Electronic Instrumentation. No. J211/1, July 2007.

[4] S. Xu, R. Dong, G. Lu, and T. X. Yu. Collision and rebounding of circular rings on rigid target. International Journal of Impact Engineering, Vol.79, No.3 (2014), P14-21.

[5] Edwin L. Fasanella and E. Karen. Best Practices for Crash Modeling and Simulation. NASA/TM-2002-211944. U.S. Army Research Laboratory. 2002.

[6] Y. Wang, et al. Experimental and numerical study of water impact investigations for aircraft ditching analysis. WCSMO-11, $7^{\text {th }}-12^{\text {th }}$ June 2015, Sydney Australia. 